\title{
Article \\ Compact Low-Cost Filter for 5G Interference Reduction in UHF Broadcasting Band
}

\author{
Oscar Fernández, Tomás Fernández (D) and Álvaro Gómez *(D) \\ Engineering of Communications Department, University of Cantabria, 39005 Santander, Spain; \\ fernanos@unican.es (O.F.); fernandt@unican.es (T.F.) \\ * Correspondence: gomezal@unican.es
}

check for updates

Citation: Fernández, O.; Fernández,

T.; Gómez, Á. Compact Low-Cost

Filter for $5 \mathrm{G}$ Interference Reduction in UHF Broadcasting Band. Electronics 2021, 10, 974. https://doi.org/ 10.3390/electronics10080974

Academic Editors: Christos J. Bouras and Gyu Myoung Lee

Received: 15 March 2021

Accepted: 16 April 2021

Published: 19 April 2021

Publisher's Note: MDPI stays neutral with regard to jurisdictional claims in published maps and institutional affiliations.

Copyright: (c) 2021 by the authors. Licensee MDPI, Basel, Switzerland. This article is an open access article distributed under the terms and conditions of the Creative Commons Attribution (CC BY) license (https:// creativecommons.org/licenses/by/ $4.0 /)$.

\begin{abstract}
The allocation of part of the UHF band to $4 \mathrm{G}$ and $5 \mathrm{G}$ services has generated the appearance of channel interferences over the digital terrestrial television frequency band. In order to reduce these interferences, this work presents a novel and efficient band-stop filter implemented using microstrip technology. The filter, designed with rectangular split-ring resonators etched in the ground plane, provides a cutoff frequency above channel $48(694 \mathrm{MHz})$, a high roll-off rate of $44 \mathrm{~dB}$ in $56 \mathrm{MHz}$ and a rejection bandwidth of $250 \mathrm{MHz}$ that covers the upper UHF band occupied by $4 \mathrm{G}$ and $5 \mathrm{G}$ with rejection levels close to $35 \mathrm{~dB}$. The filter is manufactured entirely over a printed circuit board without lumped elements to reduce production costs, fine tuning after the assembly stage and maintenance. Moreover, it presents a compact subwavelength size of only $0.07 \lambda \times 0.17 \lambda$ to facilitate installation, whether at the input of the TV terminal or integrated with the balun at the rooftop antenna.
\end{abstract}

Keywords: 5G filter; digital dividend; digital terrestrial television; split-ring resonator

\section{Introduction}

The analog television switch-off and its replacement by the digital terrestrial television (DTT) started a spectrum reassigning of the UHF band (470-862 MHz). The use of this band by a more spectrum-efficient broadcasting technology, joined with good propagation conditions, motivated the release of the upper part of the UHF V band [1,2] and its allocation to $4 \mathrm{G}$ long-term evolution (LTE) and 5G cellular mobile systems.

From the point of view of DTT, the handicap of the coexistence of LTE and DTT technologies in such close frequency bands is the apparition of interferences on the DTT signal and the overloading of DTT receivers [3-5].

Different studies [6-14] analyzed the compatibility of DTT and LTE and the impact of LTE interferences on DTT's signal quality. They presented values of the ratio required between the DTT signal and the LTE interference to guarantee a certain quality level at the DTT receptor (i.e., protection ratios). Three different DTT reception scenarios were considered: fixed outdoor, portable indoor and mobile. In these scenarios, the interference level depends on the distance between the LTE emitter and the DTT receiver, as well as other factors such as the DTT technology [8,15-19], the DTT tuner technology [20,21], DTT receiver desensitization [22], the LTE traffic load [7,8,23] or even LTE channelization [7,8].

Several works [11-14,24] proposed several approaches to minimize this problem. A straightforward solution is increasing the frequency distance between the LTE interfering signal and the desired DTT channel. Different analysis [20,21,25] showed that lower protection ratios were needed when interference and DTT signals were further away in frequency. However, UHF channel 48 and the lower part of the LTE uplink are only separated by a $9 \mathrm{MHz}$ guard band (GB). Increasing this GB can be a solution, but with important policy limitations and the counterpart of scarifying some part of the spectrum, which does not represent a spectrally efficient solution [8].

We have to consider that the farther the interference emitter, the higher the signal-tointerference ratio. Thus, guaranteeing a certain minimum physical distance between the 
LTE emitter and the DTT receiver should be a solution that would minimize interferences. For example, the authors of $[7,8]$ proposed, for indoor portable scenarios, separation distances between 6 and $8 \mathrm{~m}$ to reach the required protection ratio (i.e., assure the received signal quality). The handicap of this solution is that LTE user equipment (UE) is mainly employed by moving users not aware of how near or far they are from the DTT receiver.

A third option to solve this problem is filtering the LTE interferences. This filtering can be done at the antenna, properly designed with a bandwidth limited to the DTT bandwidth $[24,26]$. This is a good solution for new installations. However, for already deployed installations, it can be expensive since it requires the antenna's replacement. A better option is LTE filtering at the DTT antenna output or even at the receiver input. This in-line insertion of anti-LTE filters is the most simple and practical solution [6-8,11-14,24,27], since it enables the reuse of existing infrastructure (e.g., the antenna and receiver). The authors of [24] presented an overview of different existing filter technologies for individual or communal antenna systems. In $[3,4,7,28]$, different commercial anti-LTE filters manufactured through traditional techniques were analyzed: discrete inductances and capacitances, ceramic resonators and cavity filters.

In this paper, we opted for a different design and manufacture method, taking into account not only the technical aspects of its filtering response, but also other economic and technical benefits related with the manufacturing, installation and maintenance. The proposed filter is based on microstrip technology, a mature technology that facilitates the design and manufacture of economic filters in a reliable way. The traditional techniques for microstrip filter design (e.g., coupled lines, gaps, high-low impedance contrast) are not valid in these frequency bands because of their large physical dimensions. Moreover, the quality factor of the commercial substrates in these frequency bands is not high enough to obtain the desired rejection levels with these topologies. Alternative filter design techniques have gradually appeared, modifying the strip shape, nonuniform transmission lines [29,30] or the ground plane, etching continuous [31] or periodic patterns [32] (electronic band gaps (EBG)). In both cases, the resultant effect is the modification of the characteristic impedance. However, one common handicap of these techniques is that they provide electrically large structures.

Metamaterials represent a good alternative to overcome this handicap. Although they are usually periodic structures too, their compact design (tenths of wavelengths $-\lambda$ ) provides smaller unit cells than EBG. The split-ring resonator (SRRs) and its complementary version, the CSRR, are two of the most representative metamaterial structures [33]. The SRR and CSRR, properly excited, provide negative permeability and permittivity, respectively. This behavior, joined with its compact size $(\lambda / 20)$, makes SRR or CSRR suitable for the design of filters when placed near the microstrip line [34,35].

In this work, the authors present the design, characterization and experimental verification of an anti-LTE filter based on microstrip technology combined with CSRRs. One of the contributions of this paper is the analysis of some issues of the coupled CSRR microstrip filters not previously analyzed, such as the use of multi-ring CSRR or the influence of the CSRR shape on the filtering response. CSRRs are generally presented with circular or square shapes, but to the authors' best knowledge, the ratio between the ring side lengths and the relative orientation of rectangular CSRRs with respect to the microstrip line are not analyzed elsewhere.

Another important contribution of this paper is its immediate applicability in 5G interference filtering for commercial purposes. Thanks to the use of metamaterial particles, this band-stop filter reduces the channel interferences coming from the upper UHF band allocated to 4G and 5G (694-862 MHz) with a high roll-off rate and large rejection levels.

Compared with existing anti-LTE filters $[3,4,7,28]$, the proposed filter presents several benefits. First, compared with LC filters, it avoids the microphony effect thanks to its integrated design without lumped elements. In this way, it avoids variations of the electric response of the filter due to mechanic vibrations requiring less maintenance. This lack of lumped elements also avoids any additional adjustment to compensate deviations 
in the cutoff frequency or in the insertion losses, due to the tolerances of the lumped components (i.e., the filter manufacturing is cheaper). Second, compared with cavity filters, the compact filter size (due to the small dimensions of the CSRR) facilitates its installation as an independent device connected to the input of the TV receptor or integrated in the PCB joined to the antenna balun. In both cases, due to its design, it allows DC current bypass to feed, if necessary, an active UHF antenna.

\section{Microstrip Filter with CSRRs}

The proposed filter merges a microstrip transmission line with modified CSRRs to develop compact band-stop filters in a simple and economical way. The operation principle of the filter is based on the negative permittivity response of the CSRR [33]. When excited with an axial electric field provided by the microstrip line, the CSRR behaves as an electric dipole with a negative effective permittivity $\varepsilon_{r}$ [36]. Near the resonance frequency, with $\varepsilon_{r}<0$, the propagation is inhibited, providing the required band-stop filter behavior. Here, we propose the use of rectangular resonant split rings (Figure 1a), as this geometry allows us to group in a smaller surface a higher number of CSRRs than the circular one and in a more suitable position than the square one. In each CSRR, the rings are defined by the side lengths $l_{l}$ and $l_{t}$ of the outer ring, the gap $g$, the ring width $c$ and the separation between rings $d$. In the filter (Figure $1 \mathrm{~b}$ ), the microstrip line width is $w_{75 \Omega}$ and the separation between consecutive CSRRs is $s_{1}$. Rogers RO4003C has been chosen as the substrate, with its main electrical parameters being $\varepsilon_{r}=3.55$ and $\tan \delta=0.0027$ with a $1.524 \mathrm{~mm}$ high and $35 \mu \mathrm{m}$ thick copper cladding. Table 1 presents the values of the geometrical parameters of the filter shown in Figure 1a, used to perform the different simulations and to construct the final prototype. The simulations were carried out with the commercial electromagnetic software Dassault Systèmes CST Studio Suite [37] using the frequency domain solver (finite element method).

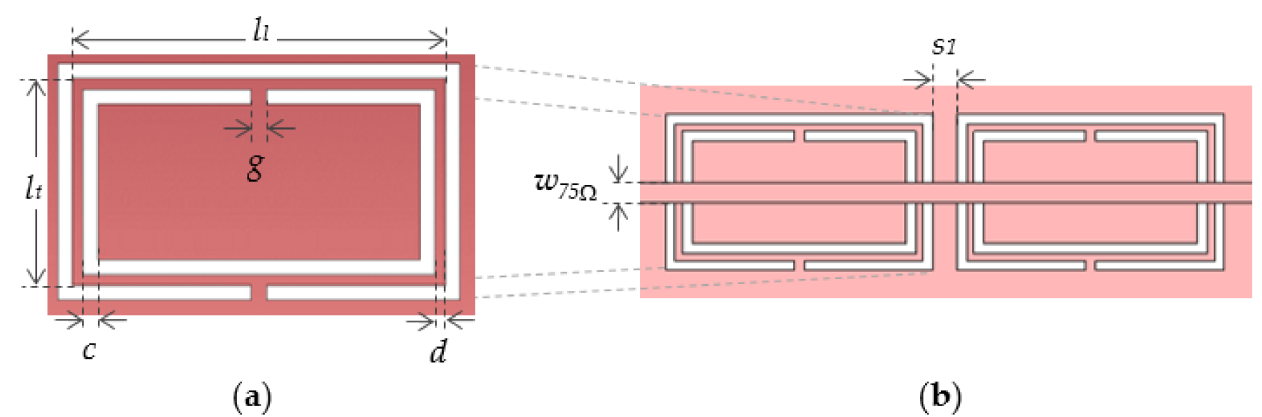

Figure 1. Schematic of the (a) two-ring CSRR and its geometrical parameters and (b) the preliminary version of the filter with two CSRR elements etched on the ground plane (the substrate is removed for better visualization).

Table 1. Numerical values of the geometrical parameters of the filter displayed in Figure 1a.

\begin{tabular}{cccc}
\hline Parameter & Value $(\mathbf{m m})$ & Parameter & Value $(\mathbf{m m})$ \\
\hline$l_{l}$ & 21.30 & $c$ & 0.40 \\
$l_{t}$ & 11.30 & $s_{1}$ & 0.50 \\
$g$ & 0.30 & $s_{2}$ & 3.00 \\
$d$ & 0.30 & $w_{75 \Omega}$ & 1.63 \\
\hline
\end{tabular}

In this section, we analyze the preliminary version of the filter presented in Figure $1 \mathrm{~b}$. The equivalent circuit model of a CSRR-coupled microstrip filter was presented and validated in [38,39]. Each section of the filter can be modeled as a lumped-element circuit (see Figure 2a), where the microstrip line is modeled through $L, C$ and the CSRR by means of $L_{x}$ and $C_{x}$. Moreover, the electric field coming from the microstrip line that excites the CSRR is modeled through the capacitance $C_{e}$ (i.e., coupling between the line and resonator). Meanwhile, the coupling between the adjacent CSRRs is modeled through $C_{M}$. In this paper, the 
circuit model is used as a qualitative tool to identify and understand key aspects of the circuit model. In fact, the model does not take into account the dielectric or ohmic losses. We have identified three key issues that will be analyzed in the following subsections: the CSRR design, the coupling between the line and CSRR and the coupling between CSRRs.

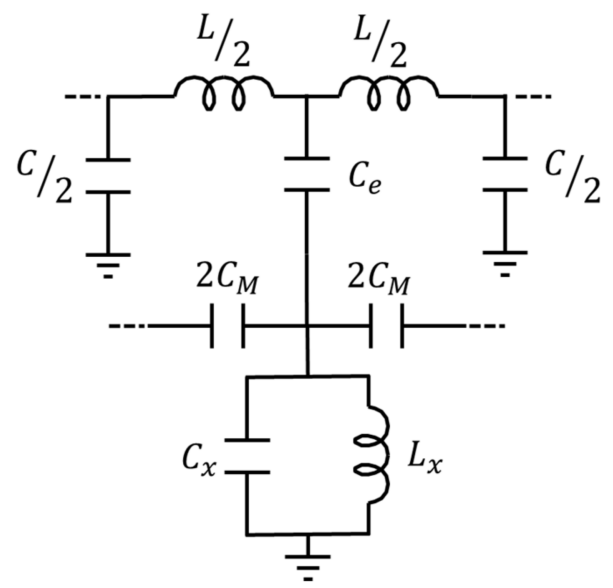

(a)

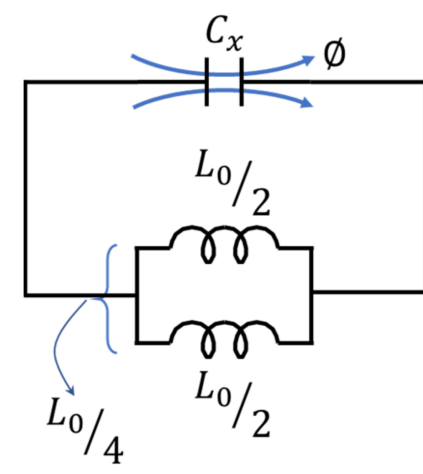

(b)

Figure 2. (a) Equivalent circuit model of one section of the CSRR-coupled microstrip filter [38]. (b) Equivalent circuit model of a two-ring CSRR [33].

\subsection{CSRR Design}

The authors of [33] presented the equivalent circuit model of a two-ring CSRR as the combination of an inductor $L_{x}$ with a capacitance $C_{x}$ (Figure $2 \mathrm{~b}$ ). The resonant frequency of the CSRR $f_{0}$ is obtained through Equation (1). The current distribution in the ground plane in the quasi-static limit at $f_{0}$ is presented in Figure 3. At this frequency, in each half of the CSRR-divided following the symmetry axis drawn in Figure 3-the current flows through the metallic strip, as indicated by the red arrows, from one gap to the other:

$$
f_{0}=\frac{1}{2 \pi \sqrt{L_{x} C_{x}}}
$$
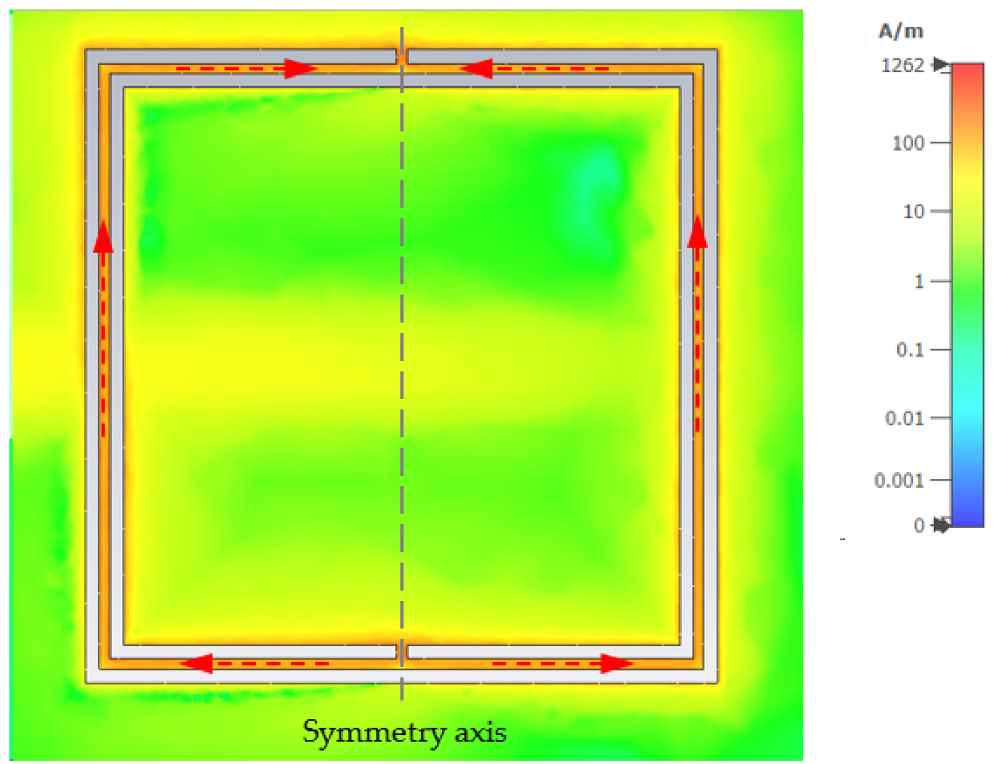

Figure 3. Surface current distribution on the ground plane around a two-ring CSRR. 
The values of these $\mathrm{L}$ and $\mathrm{C}$ elements depend on the CSRR dimensions. The slot width $c$ governs the distributed capacity, while the separation between slots $d$ controls the distributed inductance. In both cases, the total capacitance and inductance depend on the ring size (i.e., $l_{l}$ and $l_{t}$ ). Thus, the reduction of the resonance frequency can be achieved by means of its geometrical parameters, mainly by placing rings closer within the limits of the etching machine or making them larger. The handicap of this last method is clear; a lower resonance frequency requires a larger CSRR. In this paper, we follow a different and novel method to reduce the resonance frequency without increasing the CSRR size by means of increasing the total inductance $L_{x}$. Bilotti et al. [40] showed that inserting more rings in an SRR increased the total capacity. In our CSRR case, we propose etching a larger number of rings (Figure $4 a$ ) to increase $L_{x}$. Following the Babinet principle [41], the total inductance and capacitance can be obtained from those presented in [40]. For a better understanding of the influence of the inductances in this multi-ring CSRR, we present in detail the modeling of the individual inductances and its equivalent circuit model.

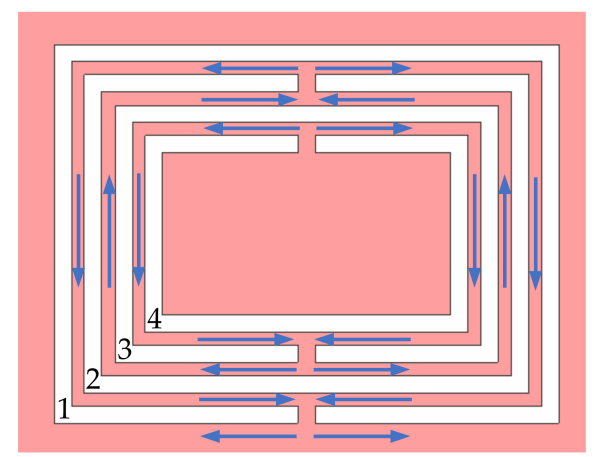

(a)

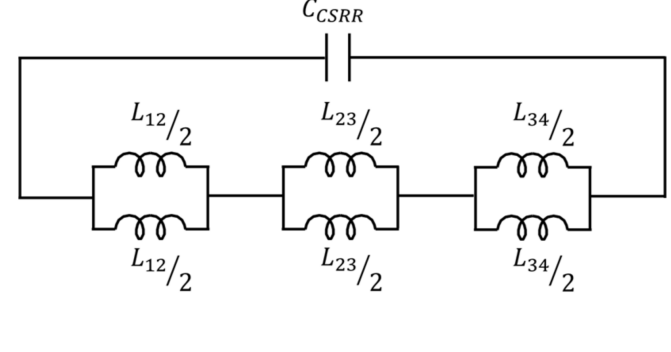

(b)

Figure 4. (a) Multiple CSRR with $N=4$ rings. The blue arrows reflect the current directions at the transmission zero frequency. (b) Equivalent circuit model of the multi-ring CSRR.

In Figure $2 \mathrm{~b}$, the inductances $L_{0} / 2$ model the two metallic strips formed between the ring slots that join both "gaps". These inductances are in parallel, and thus the total inductance $L_{x}=L_{0} / 4$. In a similar way, in the multi-ring CSRR (Figure 4a), the inductance of a pair of consecutive rings, such as rings 1 and 2, is modeled by two inductances in parallel $L_{12} / 2$. In turn, this pair of inductances is in series with the one formed by the next pair of rings ( 2 and 3 ), named $L_{23} / 2$ and so on successively. Thus, the total inductance in the equivalent circuit model of a multi-ring CSRR, presented in Figure $4 b$, is formed by the series combination of the inductances of each pair of consecutive rings. Equations (2) and (3) represent the inductances of the first pair of rings ( 1 and 2 ) and between the rings $n-1$ and $n$, wherein $L_{p u l}$ represents the inductance per unit length a coplanar strip line [33].

$$
\begin{gathered}
L_{12} / 2=\left(l_{l}-d+l_{t}-d\right) L_{p u l} \\
L_{(n-1) n} / 2=L_{12}-2[(n-1) d+(n-1) c] L_{p u l}
\end{gathered}
$$

The total inductance (Equation (4)) shows that a CSRR with a higher number of rings presents stronger inductive coupling. However, although the total inductance depends on the number of rings $N$, as the rings get smaller (i.e., larger $N$ ), their inductances are lower and contribute in a smaller proportion to the total inductance $L_{x}$ :

$L_{x}=\sum_{n=1}^{N}\left(L_{12}-2[(n-1) d+(n-1) c] L_{p u l}\right)=N \times L_{12}-2 \sum_{n=1}^{N}[(n-1) d+(n-1) c] L_{p u l}$

Figure 5 shows the $3 \mathrm{~dB}$ cutoff frequency $f_{c}$ of the filter, with CSRRs composed by a number of rings ranging from 2 to 8 and two geometries, square rings and rectangular rings. From these results, we could observe a downward frequency shift of about $180 \mathrm{MHz}$ 
in the filter response when increasing the number of rings from 2 to 6 . This effect was equivalent to a $45 \%$ reduction of the area required by a two-rectangular ring CSRR with a ratio between ring sides $r=l_{l} / l_{t}=1.9$. It is worth mentioning that with more than six rings, the response did not present significant changes. In these situations, the inner rings were much smaller than the outer ones and presented a low inductance that contributed very little to the total inductance $L_{x}$. Thus, for a given cutoff frequency, a number of rings between 4 and 6 is recommended to obtain smaller CSRRs. With this consideration, we selected CSRRs with four rings because the size reduction obtained with the six-ring CSRR of less than $10 \%$ was not significant enough in comparison with the possible additional tolerance errors that could be introduced when etching two additional rings.

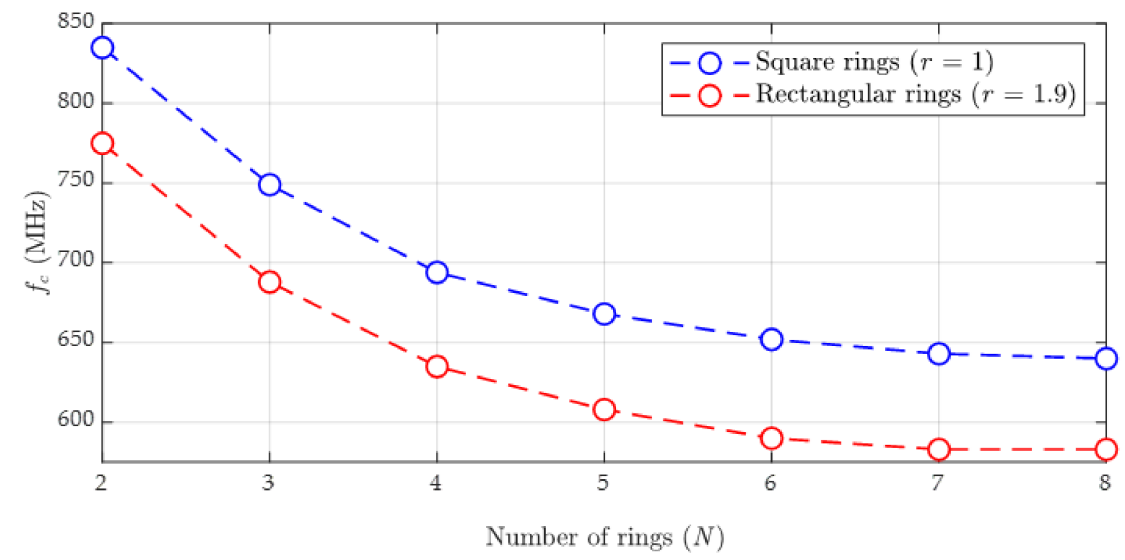

Figure 5. Cutoff frequency as a function of the number of rings in the CSRR. The blue line represents the square rings $(r=1)$, and the red line represents the rectangular rings $(r=1.9)$.

Due to this miniaturization of the CSRR, we could combine a higher number of elements or decrease the area required to implement the circuit. In fact, the number of CSRR elements that the filter includes has a great influence over its frequency response and its filtering capabilities. We can observe in Figure 6 that the increase of the element number did not modify the filter frequency cutoff frequency $f_{c}$ but enlarged the band-stop bandwidth (i.e., increasing the number of stages provided higher rejection levels). The use of four elements was a good trade-off between a convenient filter response and its size.

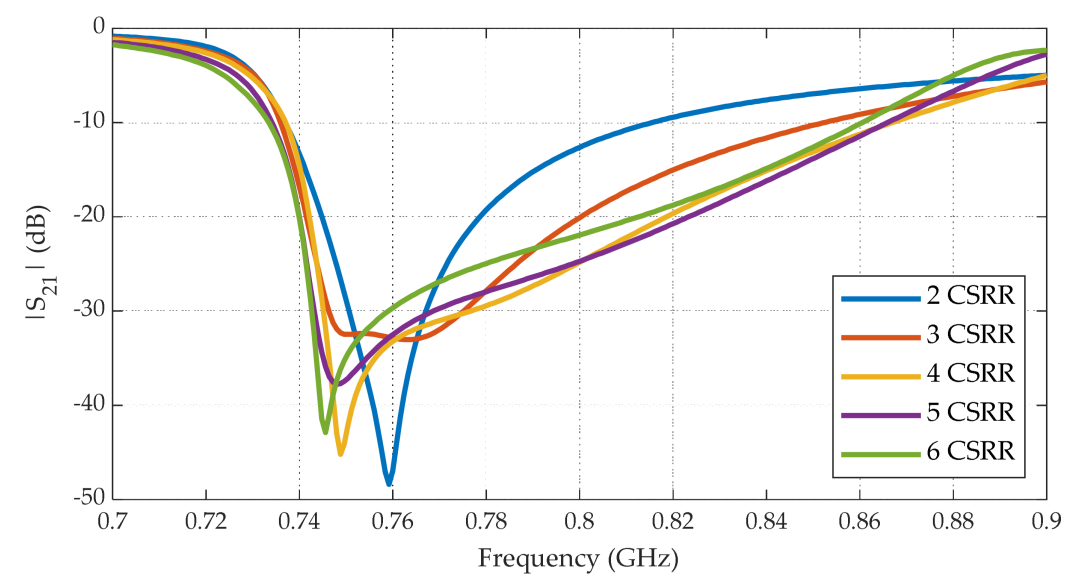

Figure 6. Filter response with a different number of elements (CSRR) with ratio $r=0.5$. Low coupling between CSRRs is considered with separation $s_{1}=10 \mathrm{~mm}$.

\subsection{Coupling between the Microstrip Line and CSRR}

When the reactance of the branch formed by $C_{e}$ in series with the $L_{x} C_{x}$ circuit (Figure 2a) is zero, the transmission of the signal is derived to the ground so there is 
no propagation of the signal to the output, thus providing a transmission zero. It is easy to find that the transmission zero occurs at the frequency $f_{z}$, as described in [38]:

$$
f_{z}=\frac{1}{2 \pi \sqrt{L_{x}\left(C_{x}+C_{e}\right)}}
$$

On inspecting Equation (5), it is clear that this frequency does not only depend on the CSRR itself but also on the coupling between the line and the resonator, modeled by the capacity $C_{e}$ in the equivalent circuit model of Figure 2a. This coupling can be modified by either changing the distance between the line and the CSRR (i.e., the substrate width) or changing the material between both metalizations [42].

In this paper, we propose a novel method that consists of the use of tailored rectangular rings to modify the line-CSRR coupling. In CSRR-based microstrip filters, the rings are etched in the ground plane and centered under the microstrip line, where the electric field is confined [33]. In contrast to circular or square rings, we propose the use of rectangular rings tailored and shaped to be placed just in the areas with higher electric field values. To evaluate the advantage of using rectangular rings, we analyze the influence of the ratio between their ring sides $r=l_{l} / l_{t}$ on the filter response but maintain a constant perimeter length of the rings (i.e., $2\left(l_{l}+l_{t}\right)=$ constant).

Figure $7 a$ presents the module of the $S_{21}$ parameter for different side ratios. It is important to note that the ratio value modified the orientation of the CSRR with regard to the microstrip line, as shown in Figure $7 b-d$. When the ratio was lower that one, the CSRR rings were placed with the shorter side parallel to the transmission line. This geometry enclosed a small surface underneath the line but also an area far from the line. Thus, very different electric field levels were present along the surface enclosed by the rings. However, for $r>1$ (i.e., rectangular rings placed with the longer side parallel to the transmission line), the area of the CSRR was mainly located in the area underneath the microstrip line, where the highest values of the electric field were present. With this ring shape modification, a higher coupling between the microstrip line and the CSRR was expected (i.e., an increment of $C_{e}$ ), and thus, following Equation (2), a lower $f_{z}$. This fact can be checked in Figure 7 where, at a first glance, we can observe that when increasing the ratio, the transmission zero frequency shifted downward. Moreover, on inspecting Figure 7 in detail, we can observe that this variation was not linear with $r$; in fact, as we can see in Figure 8, the cutoff frequency presented an exponential decay. As an example, modifying the ratio $r$ from 1 to 0.5 increased the value of $f_{c}$ by about $80 \mathrm{MHz}$, while a variation of $r$ from 1 to 1.9 resulted in a decrease of $50 \mathrm{MHz}$.

From a practical point of view, the proposed geometric arrangement and its relative orientation can be also seen as a way of miniaturizing the filter size. For example, the effect of changing $r$ from 1 to 1.9 was equivalent to reducing the perimeter of the square CSRR by about $8 \%$. Here, it is worth to mention that increasing $r$ also enlarged the filter size, so it is important to select a trade-off between the stopband width and the filter size.

On the basis of these results, the selection of the most suitable ratio value is key for defining the stopband bandwidth, the cutoff frequency and the physical size of the filter. Ratios between 1.5 and 2 are recommended to obtain a wide stopband bandwidth and a low cutoff frequency.

\subsection{Coupling between the CSRRS}

A third issue that has to be considered in the CSRR microstrip filter design is the coupling between the resonators. The results of previous sections were obtained with the resonators distanced far enough $(10 \mathrm{~mm})$ to produce a negligible coupling between them. However, from a geometric viewpoint, a large separation also requires a large filter size, something incompatible with the objective of a compact design. In this subsection, we analyze the influence of the inter-resonator coupling on the filtering response by modifying the distance between CSRRs. Moreover, we also evaluate the dependence of this coupling on the length of the CSRR side facing the neighboring CSRR. Thus, the influence of the 
aspect ratio of the CSRR is also analyzed, considering three different ratios: 0.5, 1 and 1.9. Figure 9 shows the module of the $S_{21}$ parameter for three different coupling levels: high coupling with $s_{1}=0.5 \mathrm{~mm}$, moderate coupling with $s_{1}=5 \mathrm{~mm}$ and negligible coupling with $s_{1}=10 \mathrm{~mm}$ for three different ratios: $r=0.5$ (a), 1 (b) and 1.9 (c). We observed that the main impact of the inter-resonator coupling was reflected in the stopband bandwidth. As the CSRRs were closer, the cutoff frequency kept constant, but the other end of the stopband shifted to higher frequencies, making the stopband wider. From an inter-CSRR coupling point of view, we observed that the ring ratio only affected small ratios $(r<1)$ and small distances $\left(s_{1} \leq 1 \mathrm{~mm}\right)$. In the example in Figure 9a, with $r=0.5$, the stopband for $s_{1}=0.5 \mathrm{~mm}$ was modified with respect to the other CSRR separations.

Based on these results, separation between CSRRs is a good way of controlling not only the total filter size but also the stopband bandwidth without degrading other parameters, such as the cutoff frequency or insertion losses. Special care should be taken into account when using the combination $r<1$ and $s_{1} \leq 1 \mathrm{~mm}$.

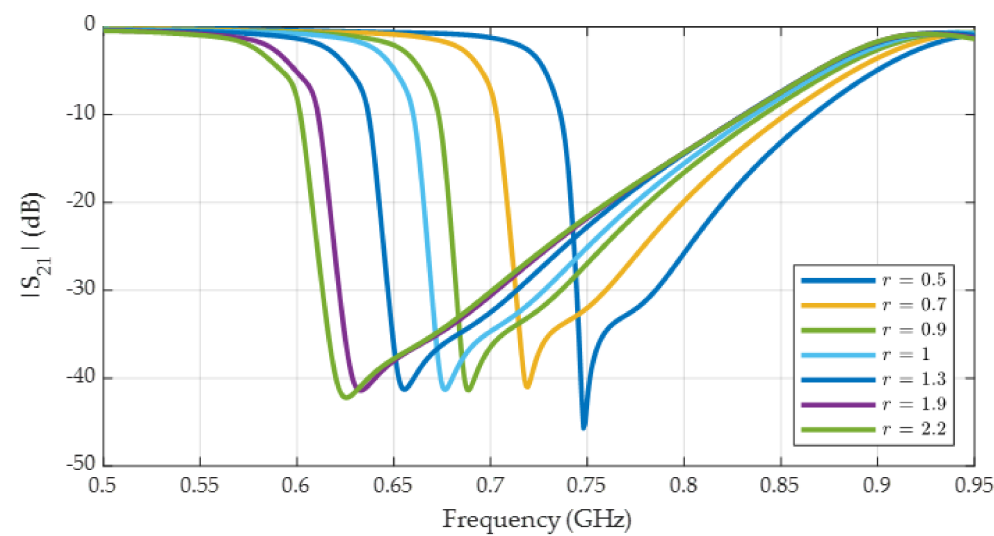

(a)

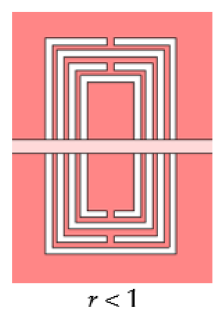

(b)

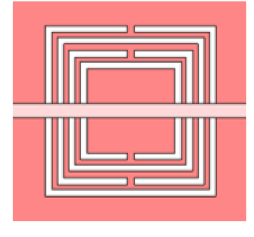

$r=1$

(c)

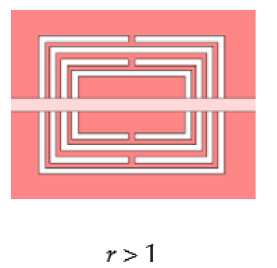

(d)

Figure 7. (a) Module of $S_{21}$ as a function of the ratio between ring sides $(r)$ where the separation $s_{1}=10 \mathrm{~mm}$ and $N=4$. Below that is graphical representation of the orientation of the CSRR with regard to the microstrip line for (b) $r<1$, (c) $r=1$ and (d) $r>1$.

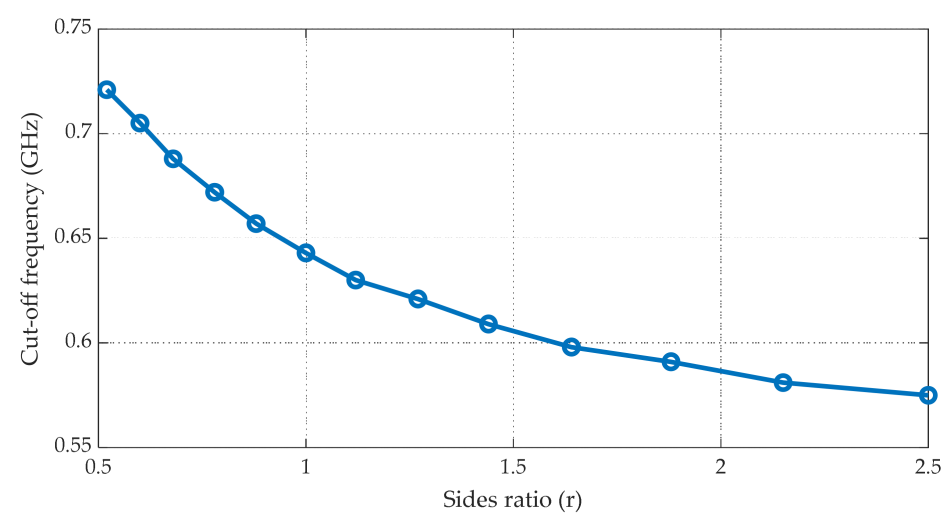

Figure 8. Cutoff frequency as a function of the ratio between the ring sides $(r)$, where the separation $s_{1}=10 \mathrm{~mm}$ and $N=4$. 


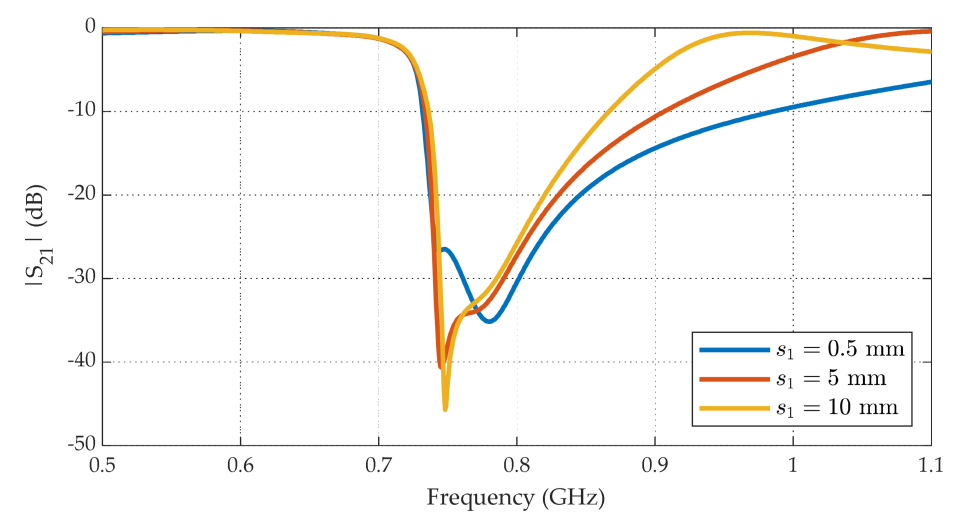

(a)

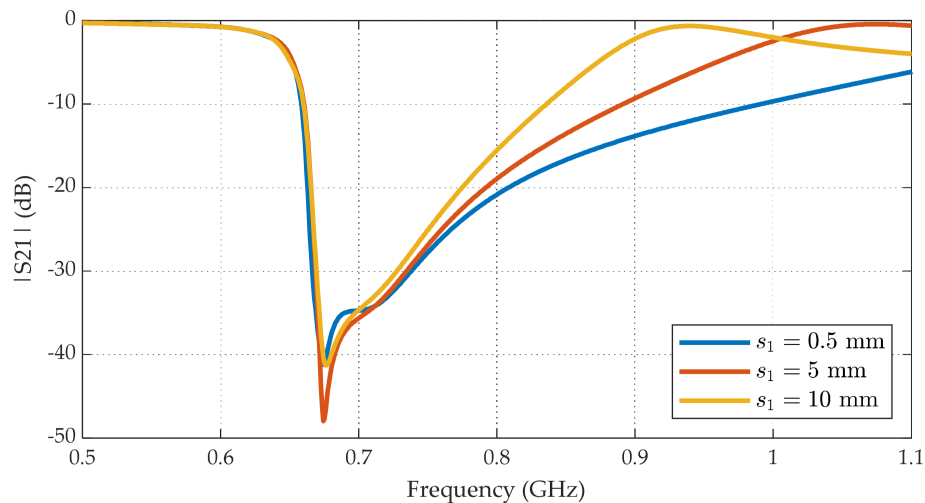

(b)

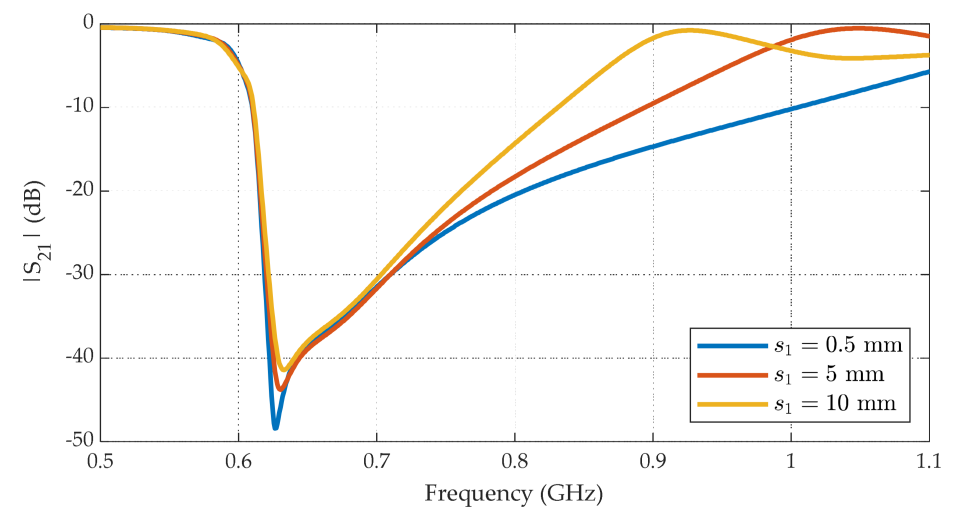

(c)

Figure 9. Module of $\mathrm{S}_{21}(\mathrm{~dB})$ of the filter with four CSRRs formed by four rectangular rings with different ratios: (a) $r=0.5$, (b) 1 and (c) 1.9. For each ratio, three different coupling levels were considered: high coupling $\left(s_{1}=0.5 \mathrm{~mm}\right)$, moderate coupling $\left(s_{1}=5 \mathrm{~mm}\right)$ and negligible coupling $\left(s_{1}=10 \mathrm{~mm}\right)$.

\section{Compact CSRR Anti-LTE Filter}

The proposed filter was designed to remove the unwanted signals generated by $5 \mathrm{G}$ technology that use the 694-862 MHz UHF band since the implantation of the first and second digital dividends. Taking this into account, the filter design objectives were the following: a $3 \mathrm{~dB}$ cutoff frequency of the filter at the end of channel 48 (this is $694 \mathrm{MHz}$ ), a sharp cutoff response (high roll-off rate) and a rejection band above $694 \mathrm{MHz}$ of at least $168 \mathrm{MHz}$, with rejection levels of about $30 \mathrm{~dB}$. In addition, the filter should be physically small to facilitate its installation on the DTT antenna, rooftop or portable, or at the input of the DTT receiver.

Based on these prerequisites and on the analysis presented in the previous section, we designed a filter with four CSRRs, each formed by four rectangular rings with a ratio 
$r=1.84$. This proportion between the side lengths placed the rings with the long sides parallel to the strip line. To reduce the length of the filter, we modified the layout of the transmission line, transforming the straight microstrip line into a U-shaped line as presented in Figure 10a. The values of all the geometrical parameters are presented in Table 1. For the experimental characterization, we manufactured several samples (Figure 10b-c) by a laser etching technique with an LPKF Protolaser S, with an etching resolution of $2 \mu \mathrm{m}$. All the measurements were carried out using an Agilent N3383A PNA Series network analyzer from $400 \mathrm{MHz}$ to $1100 \mathrm{MHz}$ with 3201 points.

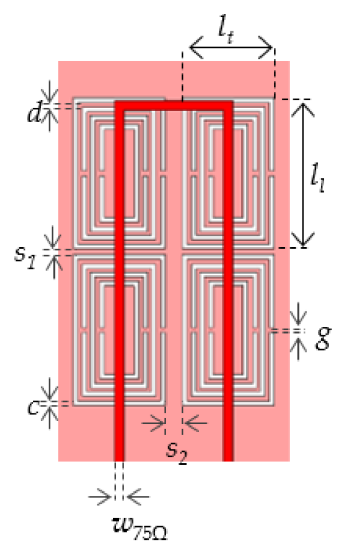

(a)

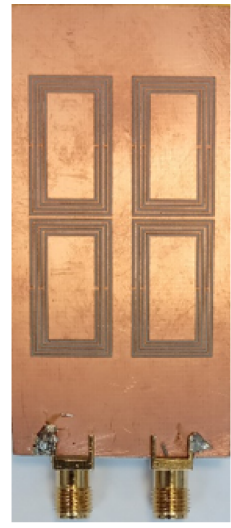

(b)

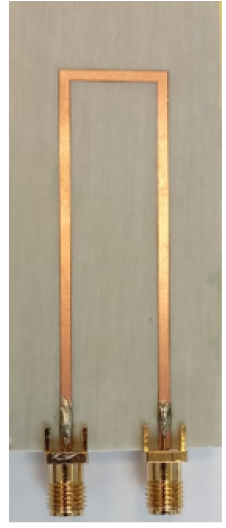

(c)

Figure 10. (a) Proposed filter (top view), not to scale, with the substrate layer hidden to facilitate the view and the manufactured filter's (b) bottom view and (c) top view.

Figure 11 shows the module of the $S_{11}$ and $S_{21}$ parameters, normalized to $75 \Omega$, comparing the results obtained from the simulations and measurements of the filter. Here, we can see a good agreement between both results with the specified cutoff frequency at $694 \mathrm{MHz}$ and a rejection band of about $250 \mathrm{MHz}$. It is important to notice that only one transmission zero was needed to obtain the required stopband to filter the UHF frequency band allocated to 4G and 5G (694-862 MHz). Furthermore, it presented a deep stopband level, with a maximum attenuation of $45 \mathrm{~dB}$ in the simulations and $35 \mathrm{~dB}$ in the measurements. In the passband, the filter introduced very low attenuation, with insertion losses lower than $0.5 \mathrm{~dB}$. Above the cutoff frequency, the module of the $\mathrm{S}_{21}$ parameter fell off rapidly, with a roll-off rate of $44 \mathrm{~dB}$ in $50 \mathrm{MHz}$. The discrepancies observed in the values of $\left|S_{11}\right|$ in the passband and $\left|S_{21}\right|$ in the stopband may have been due to possible inaccuracies in the substrate characterization at these frequencies.

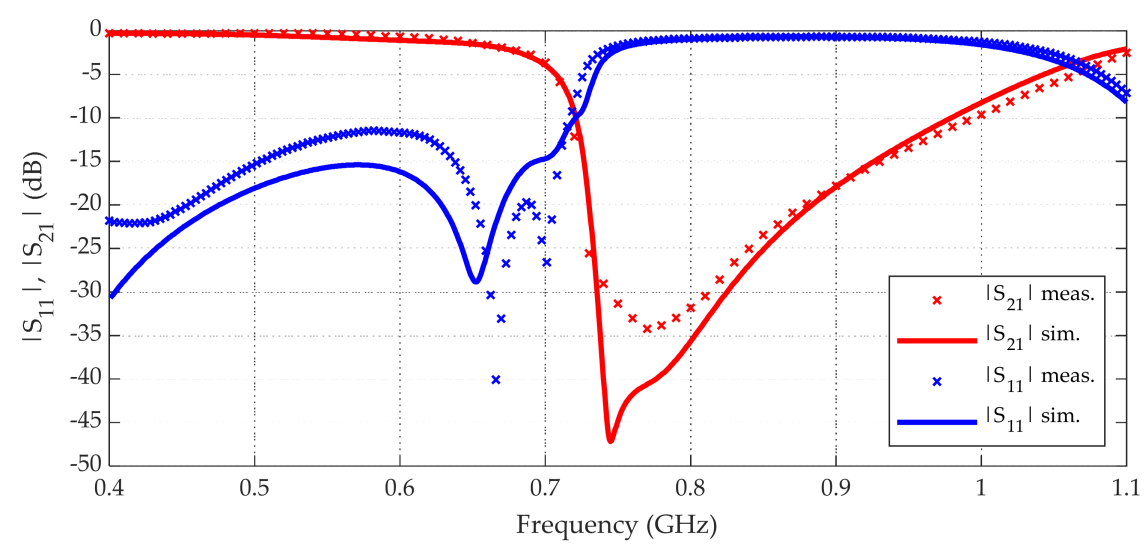

Figure 11. Module of the $S_{11}$ and $S_{21}$ parameters of the proposed filter, with a comparison between the simulations (solid) and the measurements (dots). 
Moreover, the filter size was only $35 \times 65 \mathrm{~mm}, 0.07 \lambda \times 0.17 \lambda$, with $\lambda$ being the wavelength at $600 \mathrm{MHz}$, a compact subwavelength layout for a band-stop filter with such a frequency response. This size facilitated its installation at the input of the TV receiver or its integration at the antenna sharing the PCB with the balun.

Figure 12 represents the magnitude of the surface current distributions on the ground plane at the transmission zero frequency. Although this filter presents a reciprocal behavior, in this simulation, the transmitter was connected at Port 1 (i.e., this port would be connected to the antenna), and Port 2 was connected to the receiver (i.e., it would be connected to the DTT receiver). The first CSRR - the one next to Port 1-received the unfiltered signal and thus presented the highest current amplitude levels. As the signal propagated through the stages of the filter, it was attenuated. Therefore, as can be seen in the figure, the current levels in the CSRR were rapidly decreasing, reaching values near zero at the fourth CSRR.

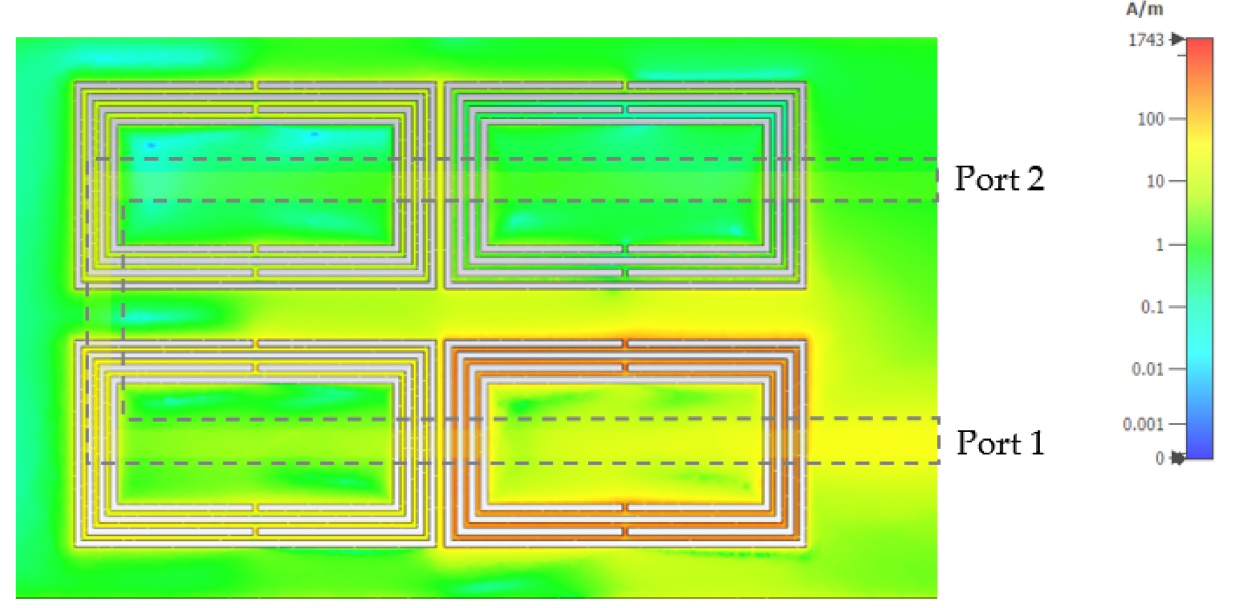

Figure 12. Current distribution in the microstrip filter ground plane at the transmission zero frequency. The dashed line represents the microstrip line.

\section{Conclusions}

In this paper, we have presented an anti-LTE filter based on microstrip technology that integrates particles with negative permittivity (CSRR). The novelty of the filter lies in its previously unreported design, with a U-shaped transmission line and rectangular CSRRs etched underneath the microstrip line in the ground plane. The importance of the relative alignment of the rectangular CSRR with respect to the microstrip line and its side length ratio for obtaining an efficient coupling has been demonstrated. In addition to the filter size reduction, the arrangement of the resonators in a rectangular lattice also enhanced the coupling among CSRRs from orthogonal directions, in contrast with the in-line distribution. This design provides a high roll-off rate and a high rejection level in a small device (only $35 \times 65 \mathrm{~mm}$ ) to facilitate its installation, whether at the input of the TV terminal or integrated with the balun at the rooftop antenna. The filter is manufactured entirely on a PCB without lumped elements to reduce the cost of manufacturing, fine-tuning and maintenance.

The validity of the proposed design was demonstrated by comparisons between the simulated and measured results, which showed good agreement. In the passband, the insertion losses were lower than $0.5 \mathrm{~dB}$, while in the rejection band, the attenuation reached up to $45 \mathrm{~dB}$. Moreover, it showed a very good roll-off rate in the transition between both bands, where $f_{c}=694 \mathrm{MHz}$. Although the filter was designed for ITU Region 1, it can be easily modified for other ITU regions.

As a result, a fully functional small-size filter has been obtained. Compared with other filter structures (e.g., lumped elements and resonant cavities) this filter provides low fabrication and tuning costs, along with response reliability due to the lack of lumped elements (i.e., component tolerance problems). Moreover, the proposed filter can be 
used either in indoor or outdoor systems without any modifications due to its bandreject response, which is its capability to allow DC bias current passthrough to the roof components. Finally, this work shows a general way to miniaturize microstrip-based filter structures, achieved using coupled resonator clusters in the ground plane along with a U-shaped transmission line in the signal plane.

Author Contributions: Conceptualization, T.F.; methodology, O.F., T.F. and Á.G.; investigation, O.F., T.F. and Á.G.; writing—original draft preparation, O.F.; writing—review and editing, O.F., T.F. and Á.G. All authors have read and agreed to the published version of the manuscript.

Funding: This research was funded in part by the Ministerio de Ciencia, Innovación y Universidades and in part by the European Regional Development Fund under Grant PGC2018-098350-B-C22.

Acknowledgments: The authors would like to also express their gratitude to all the staff of DICOM's assembling laboratory staff, as well as Eva Cuerno and Paul García for their help with the fabrication of the prototypes.

Conflicts of Interest: The authors declare no conflict of interest.

\section{References}

1. ITU. Studies on the Use of the Band 790-862 MHz by Mobile Applications and by Other Services. In Proceedings of the Final Acts of the World Radiocommunication Conference (WRC-07), World Radiocommunication Conference, Geneva, Switzerland, 2007; Resolution 749. pp. 478-479. Available online: https://www.itu.int/dms_pub/itu-r/opb/act/R-ACT-WRC.8-2007-PDF-E.pdf (accessed on 5 March 2021).

2. ITU. Final Acts of the World Radiocommunication Conference (WRC-12). In Proceedings of the World Radiocommunication Conference, Geneva, Switzerland, 2012; Available online: https://www.itu.int/dms_pub/itu-r/opb/act/R-ACT-WRC.9-2012 -PDF-E.pdf (accessed on 5 March 2021).

3. De Vita, A.; Milanesio, D.; Sacco, B.; Scotti, A. Assessment of interference to the DTT service generated by LTE signals on existing head amplifiers of collective distribution systems: A Real Case Study. IEEE Trans. Broadcast. 2014, 60, 420-429. [CrossRef]

4. ComReg LTE Filter Testing. Analysis of the Effectiveness of Currently Available LTE Filters at Reducing and Eliminating RF Overload in Masthead Amplifiers in the Presence of LTE Signals. Commission for Communications Regulation. 2012. Available online: https:/ / www.comreg.ie/_fileupload/File/compliance/12\%2009\%2007\%20Redacted\%20LTE-DTT\%20Overload\%20-\% 20Final\%20Report.pdf (accessed on 5 March 2021).

5. ECC. Measurements on the Performance of DVB-T Receivers in the Presence of Interference from the Mobile Service (Especially from UMTS); Electronic Communications Committee (ECC): Marseille, France, 2010; ECC REPORT 138; Available online: https: / /docdb.cept.org/download/04db91ba-1aef/ECCREP138.PDF (accessed on 5 March 2021).

6. Tekovic, A.; Bonefacic, D.; Sisul, G.; Nad, R. Interference analysis between mobile radio and digital terrestrial television in the Digital Dividend spectrum. Radioengineering 2017, 26, 211-220. [CrossRef]

7. Ribadeneira-Ramírez, J.; Martínez, G.; Gómez-Barquero, D.; Cardona, N. Interference Analysis Between Digital Terrestrial Television (DTT) and 4G LTE Mobile Networks in the Digital Dividend Bands. IEEE Trans. Broadcast. 2016, 62, 24-34. [CrossRef]

8. Fuentes, M.; Garcia-Pardo, C.; Garro, E.; Gomez-Barquero, D.; Cardona, N. Coexistence of digital terrestrial television and next generation cellular networks in the $700 \mathrm{MHz}$ band. IEEE Wireless Commun. 2014, 21, 63-69. [CrossRef]

9. Ofcom. Technical Analysis of Interference from Mobile Network Base Station in $800 \mathrm{MHz}$ Band to Digital Terrestrial Television; Tech. Rep.. Ofcom: London, UK, 2011. Available online: https://www.ofcom.org.uk/_data/assets/pdf_file/0019/40906/technical-report. pdf (accessed on 5 March 2021).

10. ITU-R. Measurements of Protection Ratios and Overload Thresholds for Broadcast TV Receivers. Report ITU-R BT.2215-7 (04/2018). 2018. Available online: https:/ / www.itu.int/dms_pub/itu-r/opb/rep/R-REP-BT.2215-7-2018-PDF-E.pdf (accessed on 5 March 2021).

11. CEPT. Technical Considerations Regarding Harmonisation Options for the Digital Dividend; Electronic Communications Committee (ECC), 2007. CEPT Report 23. Available online: https://docdb.cept.org/download/1eb0d908-3b2a/CEPTREP023.PDF (accessed on 5 March 2021).

12. CEPT. Report A from CEPT to the European Commission in Response to the Mandate to Develop Harmonised Technical Conditions for the 694-790 MHz ('700 MHz') Frequency Band in the EU for the Provision of Wireless Broadband and Other Uses in Support of EU Spectrum Policy Objectives; Electronic Communications Committee (ECC), 2014. CEPT Report 53. Available online: https:/ / docdb.cept.org/download/54e59598-843d/CEPTREP053.PDF (accessed on 5 March 2021).

13. CEPT. Report from CEPT to the European Commission in Response to the Mandate on the Identification of Common and Minimal (Least Restrictive) Technical Conditions for 790-862 MHz for the Digital Dividend in the European Union; Electronic Communications Committee (ECC), 2009. CEPT Report 30. Available online: https:/ / docdb.cept.org/download/3d66f450-5684/CEPTREP030.PDF (accessed on 5 March 2021). 
14. ECC. Measurements on the Performance of DVB-T Receivers in the Presence of Interference from the Mobile Service (Especially from LTE); Electronic Communications Committee (ECC), 2010. ECC Report 148. Available online: https://docdb.cept.org/download/c041 605d-e8a0/ECCREP148.PDF (accessed on 5 March 2021).

15. Polak, L.; Kaller, O.; Klozar, L.; Sebesta, J.; Kratochvil, T. Exploring and Measuring Possible Co-Existences between DVB-T2-Lite and LTE Systems in Ideal and Portable Fading Channels. J. App. Research Technol. 2015, 13, 32-44. [CrossRef]

16. Kim, D.-H.; Oh, S.-J.; Woo, J.S. Coexistence analysis between IMT system and DTV system in the $700 \mathrm{MHz}$ band. In Proceedings of the 2012 International Conference on ICT Convergence (ICTC), Jeju, Korea, 15-17 October 2012; pp. 284-288.

17. ATDI. Report for GSMA on the Coexistence of ISDB-T and LTE; Tech. Rep. W1306L4205; ATDI Ltd.: Paris, France, 2014; Available online: https:/ / www.gsma.com/latinamerica/wp-content/uploads/2014/05/lte-isdb-coexistence-study.pdf (accessed on 5 March 2021).

18. Li, W.; Chen, J.; Long, H.; Wu, B. Performance and analysis on LTE system under adjacent channel interference of broadcasting system. In Proceedings of the IEEE 12th International Conference on Computer and Information Technology (CIT), Chengdu, China, 27-29 October 2012; pp. 290-294.

19. EBU. Protection of DTT from LTE 700. EBU Fact Sheet. 2015. Available online: https://tech.ebu.ch/docs/factsheets/EBU\%20 Fact_Sheet_DTTB_protection_from_LTE700.pdf (accessed on 5 March 2021).

20. Celidonio, M.; Masullo, P.G.; Pulcini, L.; Vaser, M. Measured interference of LTE uplink signals on DVB-T channels. J. Telecom. Inform. Technol. 2015, 4, 74-85.

21. ERA. Assessment of LTE $800 \mathrm{MHz}$ Base Station Interference into DTT Receivers; ERA Technology: Leatherhead, UK, 2011; Report 2011-0351; Available online: https:/ / www.ofcom.org.uk/_data/assets/pdf_file/0027/33939/ite-800-mhz.pdf (accessed on 5 March 2021).

22. ERA. LTE Interference into Domestic Digital Television Systems; ERA Technology: Leatherhead, UK, 2010; Report 2010-0026 (Issue 2).

23. ITU-R. Planning Criteria Including Protection Ratios for Second Generation of Digital Terrestrial Television Broadcasting Systems in the VHF/UHF Bands. Report ITU-R Rec. BT.2033, 2013 (01/2013). 2013. Available online: https://www.itu.int/dms_pubrec/ itu-r/rec/bt/R-REC-BT.2033-0-201301-S!!PDF-E.pdf (accessed on 5 March 2021).

24. DVB. Study on Specification and Use of in-Line Filters to Reduce Interference in Broadcast Bands from Mobile Base Stations. DVB White Paper. 2014. Report SB2122. Available online: https:/ /dvb.org/wp-content/uploads/2020/01/DVB-InterferenceFilter-Study.pdf (accessed on 5 March 2021).

25. Liyanapathirana, R.; Gunawardana, U.; Wijesinghe, P.; Biyanwilage, S. RF interference to DVB-T reception from UMTS/LTE systems in adjacent bands. In Proceedings of the IEEE 2013 Tencon-Spring, Sydney, NSW, Australia, 17-19 April 2013; pp. 398-402.

26. Mistry, K.K.; Lazaridis, P.I.; Zaharis, Z.D.; Chochliouros, I.P.; Loh, T.H.; Gravas, I.P.; Cheadle, D. Optimization of log-periodic tv reception antenna with UHF mobile communications band rejection. Electronics 2020, 9, 1830. [CrossRef]

27. Sami, W. How Can Mobile and Broadcasting Networks Use Adjacent Bands? European Broadcasting Union Technical Review. 2011. Available online: https://tech.ebu.ch/docs/techreview/trev_2011-Q1_digital-dividend_sami.pdf (accessed on 5 March 2021).

28. Tekovic, A.; Bonefacic, D.; Nad, R.; Pidanic, J.; Nemec, Z. On performance of filter used for interference mitigation between LTE and DVB-T networks in digital dividend spectrum. In Proceedings of the 2016 International Symposium ELMAR, Zadar, Croatia, 12-14 September 2016; pp. 17-20.

29. Le Roy, M.; Perennec, A.; Toutain, S.; Calvez, L.C. The continuously varying transmission-line technique-Application to filter design. IEEE Trans. Microw. Theory Technol. 1999, 47, 1680-1687. [CrossRef]

30. Nair, N.V.; Mallick, K. An Analysis of a Width-Modulated Microstrip Periodic Structure. IEEE Trans. Microw. Theory Technol. 1984, 32, 200-204. [CrossRef]

31. Laso, M.A.G.; Lopetegi, T.; Erro, M.J.; Benito, D.; Garde, M.J.; Sorolla, M. Multiple-frequency-tuned photonic bandgap microstrip structures. IEEE Microw. Guided Wave Lett. 2000, 10, 220-222. [CrossRef]

32. Lopetegi, T.; Laso, M.A.G.; Erro, M.J.; Benito, D.; Garde, M.J.; Falcone, F.; Sorolla, M. Novel photonic bandgap microstrip structures using network topology. Microw. Opt. Technol. Lett. 2000, 25, 33-36. [CrossRef]

33. Baena, J.D.; Bonache, J.; Martín, F.; Marqués, R.; Falcone, F.; Lopetegi, T.; Laso, M.A.G.; García-García, J.; Gil, I.; Flores, M.; et al. Equivalent-circuit models for split-ring resonators and complementary split-ring resonators coupled to planar transmission lines. IEEE Trans. Microw. Theory Technol. 2005, 53, 1451-1460. [CrossRef]

34. Al-Nuaimi, M.K.T.; Whittow, W.G. Compact microstrip band stop filter using SRR and CSSR: Design, simulation and results. In Proceedings of the 4th European Conference on Antennas and Propagation (EuCAP 2010), Barcelona, Spain, 12-16 April 2010; pp. $1-5$.

35. Ponchak, G.E. Coplanar stripline coupled to planar double split-ring resonators for bandstop filters. IEEE Microw. Wirel. Components Lett. 2018, 28, 1101-1103. [CrossRef]

36. Falcone, F.; Lopetegi, T.; Baena, J.D.; Marques, R.; Martin, F.; Sorolla, M. Effective negative-/spl epsiv/ stopband microstrip lines based on complementary split ring resonators. IEEE Microw. Wireless Compon. Lett. 2004, 14, 280-282. [CrossRef]

37. Dassault Systems. CST Microwave Studio. 2020. Available online: https://www.3ds.com/products-services/simulia/products/ cst-studio-suite/ (accessed on 5 March 2021). 
38. Gil, I.; Bonache, J.; Gil, M.; García-García, J.; Martín, F.; Marqués, R. Accurate circuit analysis of resonant-type left handed transmission lines with inter-resonator coupling. J. Appl. Phys. 2006, 100, 074908. [CrossRef]

39. Marqués, R.; Ferran, M.; Sorolla, M. Metamaterials with Negative Parameters: Theory, Design, and Microwave Applications, 1st ed.; Wiley: Hoboken, NJ, USA, 2008; pp. 155-182.

40. Bilotti, F.; Toscano, A.; Vegni, L.; Aydin, K.; Alici, K.B.; Ozbay, E. Equivalent-Circuit Models for the Design of Metamaterials Based on Artificial Magnetic Inclusions. IEEE Trans. Microw Theory Technol. 2007, 55, 2865-2873. [CrossRef]

41. Falcone, F.; Lopetegi, T.; Laso, M.A.G.; Baena, J.D.; Bonache, J.; Beruete, M.; Marqués, R.; Martín, F.; Sorolla, M. Babinet principle applied to the design of metasurfaces and metamaterials. Phys. Rev. Lett. 2004, 93, 2-5. [CrossRef] [PubMed]

42. Öznazı, V.; Ertürk, V.B. A comparative investigation of SRR- and CSRR-based band-reject filters: Simulations, experiments, and discussions. Microw. Opt. Technol. Lett. 2008, 50, 519-523. [CrossRef] 